\title{
The Difference in Mass between Relativity and Quantum Mechanics, Also Novel Effects of the Axial Doppler Shift
}

\author{
Samuel Lewis Reich ${ }^{*}$, Winston G. Perera ${ }^{2}$ \\ ${ }^{1}$ Warrensville Heights, OH, USA \\ ${ }^{2}$ Case Western Reserve University, Cleveland, OH, USA \\ Email: *slrch53@gmail.com
}

How to cite this paper: Reich, S.L. and Perera, W.G. (2019) The Difference in Mass between Relativity and Quantum Mechanics, Also Novel Effects of the Axial Doppler Shift. Journal of High Energy Physics, Gravitation and Cosmology, 5, 629-637. https://doi.org/10.4236/jhepgc.2019.53035

Received: April 14, 2019

Accepted: May 19, 2019

Published: May 22, 2019

Copyright $\odot 2019$ by author(s) and Scientific Research Publishing Inc. This work is licensed under the Creative Commons Attribution International License (CC BY 4.0).

http://creativecommons.org/licenses/by/4.0/

\begin{abstract}
If a particle has a wave function or is in other ways a moving wave, it should have an axial Doppler shift. Writers on relativity do not give moving particles that. The classic equation of quantum mechanics requires that frequency and mass have the same distortion from velocity (Doppler shift). But in the common writings on relativity mass always goes up with increases of velocity, and the transverse shift of frequency always goes down with increases of velocity [1] [2] [3] [4]. Most of this is due to simplifications and errors in the Lorentz transformation, some came from being in the aether wind era originally and because accelerators are noisy. It is not valid to say because the aether axial wind averages to zero between reflections so does axial Doppler shifts. After the first reflection in the Lorentz transformation, the light from the Sun is in Earth's reference frame and there are no more Doppler shifts. Also the Michelson-Morley experiment is not all cases, and light is not the only thing deformed by velocity. The axial shift's formula has the cosine of the observation angle in it. The implications are not just quantitative but also qualitative because anything with an axial Doppler shift has different values in different directions from an observer. That is the defining property of a vector and that changes its dimensions and the dimensions of the differential relations it is in. This happens with other scalar qualities as well. That means scalars such as mass and charge are now vectors and have additional dimensions. Therefore differential equations with them have additional dimensions. This includes Faraday-Maxwell's equations and Schrodinger's equations. Also the Doppler blue shift seems to imply additional dimensions of time another way. That is the first Lorentz transformation error; the second is assumption of non-existent symmetry.
\end{abstract}

\section{Keywords}

Wave-Function, Relativity, Doppler, Mass, De Broglie, Schrodinger, 


\section{Lack of Correction}

The writers on relativity do not get corrected because the only places on Earth where there are high relativity effects are in accelerators, which are very, very noisy, and in observations where the observation angle is random (cosmic rays, radio activity). The average of axial Doppler shift over all angles is zero but if someone looks for it the variance is about one third of the maxim shift. Also Lorentz and Einstein, are academic heroes, for over 100 years, because they convinced the world that velocity changes time and space and at least qualitatively that is proven even if there is quantitate errors. Even without relativity, the two are significant in physics. Given that and the need for a powerful low noise accelerator, few experimenters will get grants to prove the existence systematic quantitative errors in relativity.

Note: Although the average of the magnitude of the axial shift over all angles is zero, the variance of the magnitude over all angles is about $1 / 3$ of the maximum magnitude. Which means it affects energy distribution functions in gases and plasmas and distribution functions in results where angles vary randomly in measurements as in the Compton effect. Also the maximum magnitude of the axial Doppler is higher than the transverse shift.

\section{Combining Axial and Transverse Doppler Shifts by Einstein's Second Postulate with Both Axial and Transverse Motion of the Moving Reference Frame}

The source of light has a reference plane indicated by no superscript. The primed values refer to the moving observer's reference plane. The observer in Figure 1 is moving with a transverse velocity $v_{t}$ to the light and with a velocity $v_{a}$ parallel to the light. In the case of a narrow beam, the velocity of the observer cannot always be aligned with that of the beam. Also, there is no reflection here to cancel $V$

$c \Delta t^{\prime}=\sqrt{\left(c-v_{a}\right)^{2}+v_{t}^{2}} \Delta t$ and $v_{r}=\left(v_{t}^{2}+v_{a}^{2}\right)^{1 / 2}$ the resultant velocity, and $v_{a}=v_{r} \cos \theta$ also $v_{t}=v_{r} \sin \theta$.

In Einstein's second postulate $\left(c=c^{\prime}\right)$, the speed of light appears to be same in all reference planes. Therefore, $c^{\prime}=\left(\left(c-v_{a}\right)^{2}+v_{t}^{2}\right)^{1 / 2}$.

Relativity researchers take the special case of no $v_{a}$ by canceling out $v_{a}$ by reflection. That becomes an error of omission if it is presented as the general case. There is no reflection in this analysis. The Michelson-Morley experiment has multiple reflections and most relativity writers say the resulting red and blue shifts cancel each other. But only the first reflection has a Doppler shift, at best. After that the light is in earth's reference frame (no more shifts). Also there still is an axial shift for the Sun's light with or without a reflection. 

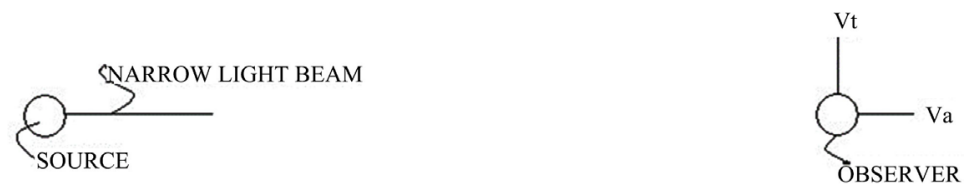

OBJECTS

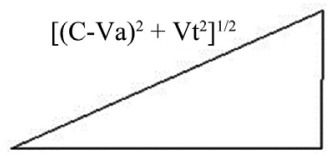

Velocity Addition

Figure 1. Graphics of Deriving the General Case of the Resultant Doppler Shift.

$$
\begin{aligned}
& c t^{\prime}=c^{\prime} t \\
& t^{\prime} / t=\left[\left(1-\left(v_{a} / c\right)\right)^{2}+\left(v_{t} / c\right)^{2}\right]^{1 / 2}=1 / K_{r}
\end{aligned}
$$

In terms of frequencies, $\omega^{\prime} / \omega=t / t^{\prime}=1 /\left[\left(1-\left(v_{a} / c\right)\right)^{2}+\left(v_{t} / c\right)^{2}\right]^{1 / 2}$ is the time contraction factor. If the direction $v_{a}$ is in the opposite direction, the time dilation factor is $1 /\left[\left(1+\left(v_{a} / c\right)\right)^{2}+\left(v_{t} / c\right)^{2}\right]^{1 / 2}$. If $v=\left(v_{a}^{2}+v_{t}^{2}\right)^{1 / 2}$, then $K_{r}=1 /\left[1-2\left(v_{a} / c\right)+\left(v_{t} / c\right)^{2}\right]^{1 / 2}$. If $v_{a}=v \cos (\theta)$, then $v_{t}=\sin (\theta)$ and $K_{r}=1 /\left[1-2(v / c) \cos (\theta)+(v / c)^{2}\right]^{1 / 2}$

This is close in numerical value to simply multiplying the axial and transverse shifts to obtain $K_{r}$

If $v_{a}=0$, the common equation for transverse Doppler shift $t / t^{\prime}=\omega^{\prime} / \omega=1 /\left(1+\left(v_{t} / c\right)^{2}\right)^{1 / 2}$ is obtained.

\section{Modified Mass and Wave Function}

\subsection{Effect of Axial Shift on Wave Functions and the Schrodinger Equation Itself}

If the wave function is mathematically a wave in space and time it has the same Doppler shift as all such waves $\left(K_{r}\right)$.

As said before the axial shift has an observation angle in it's formula that is dependent on the position of the observed relative to the observer. That means $E$ and $m$ have different values in different directions relative to the observer. Since either the observer or the observed is moving to have a Doppler shift: that angle is changing all the time, even if the observer does not translate or rotate relative to its' reference frame. Therefore $m$ and $E$ have become three dimensional vectors and Schrodinger equation becomes: 
Before (scalar $m$ and $E$ ): Schrodinger equation [5] [6]:

$$
-\left(\hbar^{2} / 2\right) \nabla^{2} \psi+m V \psi=m E \psi
$$

After (vector $M$ and $E$ ): $\quad-\left(\hbar^{2} / 2\right) \nabla^{2} \psi+m \cdot V \psi=m \cdot E \psi$

Since one needs to solve in most cases simultaneous equations in special relativity: If one wants to add quantum effects, add any equations defining the wave function.

\subsection{Effect on Mass}

$$
\text { De Broglie's wavelength }=\lambda=h / p=h /(m v)
$$

where $p=$ momentum, $v=$ group velocity and $h=$ Plank's constant

Multiplying both sides by $m v^{2} / \lambda$ results in $m v^{2}=h v / \lambda=h f$ where $f$ is frequency $v / \lambda$. Therefore, the mass of small particles $m=h f / v^{2}$. If the length (points passed) and time of the velocity (length/time) are in the same reference frame (that of the observer), they both have the same Doppler shift factor $1 / K$, where $K$ is the Doppler shift of the frequency. As a result, that velocity (invariant) has a Doppler shift $=1$, if the quantum equations are in the observer's reference frame. Therefore, the mass $(m)$ is proportional to frequency $(f)$. As a result, $\mathrm{m}$ has the same Doppler shift as $f$, which is $K$.

$$
\begin{aligned}
& m=K_{r} m^{\prime}=m^{\prime} /\left[1-2(v / c) \cos (\theta)+(v / c)^{2}\right]^{1 / 2} . \text { This is different than } \\
& m=m^{\prime} /\left[1-(v / c)^{2}\right]^{1 / 2} \text { found in relativity books. }
\end{aligned}
$$

A test of this analysis involves Schrodinger's equation [5] [6]:

$-\left(\hbar^{2} /(2 m)\right) \nabla^{2} \psi+V \psi=E \psi . \Psi, V$ (potential), $m$ (mass), and $E$ (energy) have a Doppler shift of $K_{r} \quad \nabla$ is $1 /$ distance and $\nabla$ has a shift of $K_{r}$. Therefore, the shifts' $\left(K_{I}\right)$ would cancel out in the equation. This is true only if $V, E, \nabla$ and $\mathrm{m}$ have the same Doppler shift factor $K$.

Since one needs to solve in most cases simultaneous equations in special relativity, if one wants to add quantum effects, an equation (any) solving for the wave function must be added. But attempts at full relativistic quantum mechanics tend to fail. According to most relativity scholars, $E, V$, and $m$ do not have the same (velocity distortion) Doppler shift factor as Schrodinger's equation did for $(1 /$ distance $)=($ Doperator $)$, and that is a contributing cause for the failures.

We measure mass by the force its fields exert on objects. If one has a moving atom with a distance $\lambda$ between two quantum states of a field and velocity $\mathrm{v}$, the frequency other objects see is $K v / \lambda$. This means that the energy of the atom's fields other objects see is $\mathrm{K}$ times the objects would see if the atom was still. Therefore, this paper presents $m=m^{\prime} K$ as the best estimate of how mass is distorted by velocity.

\subsection{Proposed Experimental Verification of Axial Doppler Shift on Mass}

Setup: One relativity slow beam of electrons in the $X$ direction of a low velocity 
$V_{a}$ and in the $x y$ plane a beam of electrons intersecting it at 45 degrees with a relativistic velocity $v_{b}$ and a later time at 235 degrees. All the velocities and rest masses are in the earth's reference plane.

Analysis: Then each mass as seen by the slow moving electrons is $m=m_{o} K$. Where $m_{o}$ is the rest mass and $K$ the Doppler shift factor. From section 1.0, $K_{r}=1 /\left[1-2(v / c) \cos (\theta)+(v / c)^{2}\right]^{1 / 2}$. Where $\theta$ is either the 45 degrees or the 235 degrees. The momentum after impact should verify the relations of $\mathrm{m} / \mathrm{m}_{0}$ given above. In particular the large variation with $\theta$.

\section{Analysis of a Conveyor Belt (Proof That the Axial Doppler Shift Formula Is Universal and Due to the Nature of Time and Space)}

Every $1 / \omega$ seconds, an object is placed on a conveyor belt moving with velocity $c$ (a vector) taken relative to a stationary observer, and the items are to be detected by a downward-looking detector moving above the belt with a vector velocity $V$ relative to the same stationary observer. The positive direction is a velocity moving from the source to the observer. Here in Figure 2, $c$ is used because the belt speed has the same effect as the group velocity of light in the optical Doppler effect. Let $v \cdot c=\|c\|\|v\| \cos \theta \cdot \theta$ is the angle between the two velocities. The closing velocity (the sum of the components of $c$ and $v$ moving the items toward the detector) is $c-v \cos \theta$. As a result, in one second, $[\omega(c-v \cos \theta) / c]$ items are detected. This is the same equation as the axial Doppler shift of the frequency for waves moving at speed $c$ and an observer with a velocity $v$ at an angle $\theta$ to $c$.

Likewise, the classic Doppler shift equation applies to all moving periodic items such as the conveyor example and a machine gun bullet stream, not just waves. The classic term here is axial Doppler shift. If information is encoded such that a black item is a dot and a polished metal item is a dash on the conveyor belt, the rate of Morse-coded information observed by a reflected light sensor (the detector) is changed by the same shift factor $(c-v \cos \theta) / c$ as the all other frequencies of the passing objects. Since the passing frequency of all the detectable properties of the items on the conveyor have their rates of detection shifted by the same factor, time appears to have been compressed or dilated depending on the sign of $v$. Because the axial Doppler shift formula occurs in all of the waves and many other items such as conveyor belts or a stream of bullets, the Doppler shifts are due to the geometric properties (topology) of time and space and not the physical properties of the observed items or media, except the latter determines the group velocity $(C)$ at which the conveyor belt, bullets, or waves move.

By extension, the observation rate of all of the information in the type of observations previously described was changed by the same Doppler factor formula as the aforementioned frequencies. Therefore, similar to the relativistic optical shift, the axial factor of all of the Doppler shifts is also the time dilation or compression depending on if it is a red or blue shift. All of the observations of the stream of objects or waves are compressed or dilated depending on if it is a red 


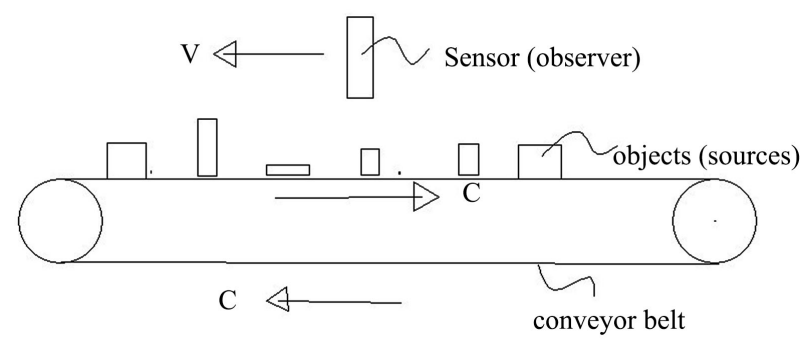

Figure 2. Sketch of a Conveyor Belt with a Doppler Shift.

or blue shift. Since the propagation velocity of light and sound is a constant independent of any Doppler shift, when time (1/frequency) is multiplied by a factor of $1 / K$, the space dimension ( $c$ /frequency) along the propagation axis is also multiplied by $1 / K$.

Relativity researchers post that only the transverse shift is the time dilation. But the aforementioned conveyor belt example indicates that it is plain that all of the detectible rate properties (1/time period properties) of waves and periodic moving objects undergo a classic axial Doppler shift. As a result, the significance of the axial shift is that it is time compression or dilation (changes in the observed length of the time segments).

Because high-energy beams are noisy for various reasons and it is impossible to control experiments on objects light years away, errors remain. Various researchers have made some corrections.

This paper describes the axial Doppler shift's significance and not the exact formulas for the magnitudes of the transverse Doppler shift $\left(K_{t}\right)$, the axial Doppler shift $\left(K_{a}\right)$, or the total Doppler shift $(K)$. Therefore, the transverse Doppler shift's magnitude is $\omega^{\prime} / \omega=K_{t}$ without defining the equation for $K_{t}$ (where $\omega$ equals the frequency of a moving source as measured moving with it and $\omega^{\prime}$ is the magnitude of $\omega$ as detected by a stationary observer). This will avoid any errors due to the difficulty in measuring light emanating from objects traveling at significant portions of light's velocity. Another difficulty in the measurement of $K_{t}$ is that for most angles, the axial Doppler shifts are much larger that the transverse Doppler shifts. [7] shows the most commonly written combination of axial and transverse shifts as simply the product of the two. $K_{a}$ represents the magnitude of the axial shift and $K=K_{a} K_{t}$ represents the magnitude of the total shift. This paper also indicates that a non-linear combination will result if one avoids assumptions in the Lorenz transformation. This paper also notes that any significant result of body forces acting on photons should be in any analysis.

\section{The Effect of the Axial Shift on Scalar Quantities with a Doppler Shift}

Time, mass, and many other scalar quantities that have a Doppler shift have an axial part of the shift that varies with the observation angle. Therefore, they vary with the positon and the velocity of the observer relative to the source in 
three-dimensional space. This means they are multi-dimensional vectors and are no longer scalars. Differential equations that have them as variables now have extra terms. A good example of this is Faraday's law.

\section{An Example of Axial Shift's Impact on Differential Laws with Scalar Variables}

One impact on Faraday's law, is that time $(t)$, now has different values in different directions. This implies that for any vector quantity $G, \Sigma_{i}^{3} \partial G_{i} / \partial T_{i} 1_{i} \quad\left(1_{i}=\right.$ unit vector in the I direction) must replace $\partial G / \partial T$ at high $(v / c)$ in differential equations. It is a time $\operatorname{grad} G$. Let $(G)$ be $\Sigma_{i}^{3} \partial G_{i} / \partial T_{i} 1_{i}$ and let $\operatorname{trad}(G)$ be the same. One is easy to type and the other is easy to write. The velocity now becomes $\Sigma_{i}^{3} \partial L_{i} / \partial T_{i} 1_{i}$ or $\operatorname{trad}(L)$, where $L$ is a length vector. Faraday's law $\operatorname{curl} \boldsymbol{E}+(1 / c) \partial \boldsymbol{B} / \partial t=0$, at high $v / c$, becomes $\operatorname{curl} \boldsymbol{E}+(1 / c) \operatorname{trad} \boldsymbol{B}=0$. This is one of Maxwell's equations, which is the only one that has $\partial / \partial t$. The other three remain unchanged.

The wave equation form is $\nabla^{2} \boldsymbol{A}-\left(1 / c^{2}\right) \partial^{2} \boldsymbol{A} / \partial t^{2}=0$, which becomes $\nabla^{2} \boldsymbol{A}-\left(1 / c^{2}\right) \Sigma_{i}^{3} \partial^{2} \boldsymbol{A}_{i} / \partial t_{i}{ }^{2}=0$. The observer sees it as $\operatorname{curl} \boldsymbol{E}^{\prime}+(1 / c) \operatorname{trad} \boldsymbol{B}^{\prime}=0$ and $\nabla^{2} \boldsymbol{A}^{\prime}-\left(1 / c^{2}\right) \Sigma_{i}^{3} \partial^{2} \boldsymbol{A}_{i}^{\prime} / \partial t_{i}^{2}=0$. To obtain the maximum effect of $K$, one must solve along the axis of maximum $K$. Since time and space have the same Doppler shift, along any axis the equation and solution has the same form in any reference. $E$ and $B$ are affected by Doppler shifts.

For example, the homogenous or traveling wave solution is $\Sigma_{i}^{3} F_{i}\left(\sin \left(k_{i} s_{i}-\omega_{i} t_{i}\right)\right)$. If the Doppler for $\omega$ is $K$, then that of $t$ is $1 / K$. Likewise, that of $k$ is $1 / K$ and that of $s$ is $1 / K$. Therefore, $\left(\sin \left(k_{i} s_{i}-\omega_{i} t_{i}\right)\right)$ is the same in all of the reference planes. Therefore, traveling wave solutions have the same form in all of the reference planes. But $F_{i}$ itself has a Doppler shift between the reference planes.

\section{How Axial Blue Shift Supplies Candidates for Bohm's Missing Dimensions}

\subsection{A Law of Time (for Use on the Blue Shift)}

Exact observations of the future are impossible because of unknown noise or multiple futures. This is because, in a universe with only one future, any group with a future observing device would try to negate undesirable and avoidable events. But in the macroscopic world with only one future, an event and its negation cannot exist at the same time. So that group could not obtain an exact observation of an avoidable and undesirable event until at the very least the event was no longer avoidable. Therefore, there exists some undocumented noise or multiple futures in the nature of time.

\subsection{Problems with Information in Blue Shifted Carrier Waves Reflected from a Repeater}

If a modulated beam of duration $G$ is reflected by a moving repeater (a mirror or 
other object absorbs it before sending a repeat) back to the source, the source sees a Doppler shift factor not just $K$ but $K^{2}$. The existence of Doppler radar proves that reflections have a Doppler shift. When a repeater moves toward both the source and the observer, the observer sees $K^{2}$ as a double blue shift. This means, if there is no built-in noise and there is only one future, information would be seen faster by the observer than it is sent by the source. That is the observer seeing information coming from the future. But from the new law of time, an observer on the source cannot see an avoidable event until the event is no longer avoidable. The existence of time travel ideas such as worm holes is also have the same problems for the same reason.

Since information can be sent by Morse code with bursts of noise, noise can be eliminated as an explanation. As said before, multiple time dimensions are at odds with the common concept of time. But the blue shift implies such dimensions. David Bohm proposed a long time ago that the probabilistic nature of quantum mechanics implies missing dimensions. The axial Doppler blue shift supplies candidate dimensions for the missing dimensions.

\subsection{Schrodinger Time Varying Equation with Multiple Time Dimensions}

[6] $i \hbar \mathrm{d} \Psi / \mathrm{d} t=H \Psi(H=$ Hamiltonian of the mass $)$.

For many time dimensions, let ${ }^{j} t$ be the value of the $j$ th time dimension and ${ }^{m} \Psi$ the wave function (probability of the value of ${ }^{w} t$ ).

Then obviously a great number of js: $i \hbar \int\left(\partial^{j}\left({ }^{w} \Psi\right) / \partial^{j} t\right) \mathrm{d} j={ }^{w} H^{w} \Psi$.

For a few js: $i \hbar \Sigma^{j} \partial^{w} \Psi / \partial^{j} t={ }^{w} H^{w} \Psi$.

\section{Summary}

The paper gave reasons for believing that:

1) Basic quantum mechanics is consistent with $m=m^{\prime} K$ where $K=$ frequency impacting the observer/frequency affecting the source $=$ Doppler shift factor. Equations of relativity have increases in velocity increasing mass but its transverse Doppler shift decreasing frequency when velocity increases.

2) The axial Doppler shift formula has an observation angle in it and the axial Doppler shift affects most physical things. Since observation angle is dependent on the three dimensional position of the observer relative to the observed that angle gives three extra dimensions (variables) to each quality affected and the Doppler shift factor $(K)$. This adds terms (dimensions) to the differential equations between physical qualities.

3) The axial Doppler shift is universal and affects all collections moving at a uniform group velocity not just waves.

4) Blue shifts seem to imply time is multidimensional.

\section{Conflicts of Interest}

The authors declare no conflicts of interest regarding the publication of this paper. 


\section{References}

[1] Einstein, A. (1955) The Meaning of Relativity. 5th Edition, Princeton University Press, Princeton, 36.

[2] Ugarov, V.A. (1979) Special Theory of Relativity. MIR Publishers, Moscow, 83-84.

[3] Fowles, G.R. (1989) Introduction to Modern Optics. Dover Publications, New York.

[4] (2016) Search “Time Dilation”. https://www.wikipedia.org/

[5] Hecht, K.T. (2000) Quantum Mechanics. Springer-Verlag, New York.

[6] Newton, R.G. (2002) Quantum Physics. Springer-Verlag, New York.

[7] Jackson, J.D. (1975) Classical Electrodynamics. John Wiley and Sons. 